\title{
MENINGIOMAS PARASSELARES: ASPECTOS NA RESSONÂNCIA MAGNÉTICA*
}

\author{
Alair Augusto S.M.D. dos Santos ${ }^{1}$, Denise Madeira Moreira ${ }^{2}$, Pedro Ângelo Andreiuolo ${ }^{3}$, \\ Fernando Barros de Oliveira ${ }^{4}$, Ricardo Tostes D. Teixeira ${ }^{4}$, Cristina Asvolinsque P. Fontes ${ }^{5}$, \\ Saul Orlando C. Correa ${ }^{4}$
}

\begin{abstract}
Resumo Foram revistos 22 casos de meningiomas parasselares avaliados por ressonância magnética em clínicas privadas nas cidades de Niterói e Rio de Janeiro, RJ. O objetivo deste trabalho é demonstrar os aspectos de imagem encontrados neste tumor. Os equipamentos utilizados tinham 0,5 e 1,0 Tesla, respectivamente, com aquisição de seqüências multiplanares ponderadas em T1 (antes e após a administração de gadolínio) e em T2. Os sintomas principais foram cefaléia e alterações visuais, tendo apenas um caso cursado com hiperprolactinemia. $O$ aspecto mais freqüente foi o de massa parasselar hipointensa em T1 e hiperintensa em T2, impregnando-se intensamente pelo gadolínio. A ressonância magnética é útil não apenas na demonstração da lesão mas, sobretudo, na avaliação do comprometimento das estruturas vizinhas, especialmente do seio cavernoso, o que explica as alteraçōes visuais encontradas.
\end{abstract}

Unitermos: Meningioma parasselar. Tumores cerebrais. Ressonância magnética.

\begin{abstract}
Parasellar meningiomas: magnetic resonance imaging findings.
We reviewed 22 cases of patients with parasellar meningiomas evaluated with magnetic resonance imaging (MRI) in private clinics of the cities of Niterói and Rio de Janeiro, RJ, Brazil. Our aim was to characterize the imaging findings in this type of tumor. MRI scanners with 0.5 and 1.0 Tesla magnets were used for the acquisition of multiplanar T1-weighted (pre- and post-gadolinium administration) and T2-weighted images. The main symptoms observed were headache and visual disturbances. Hyperprolactinemia was observed in only one patient. The most frequent imaging finding was a parasellar mass which appeared hypointense on T1-weighted and hyperintense on T2-weighted images, and enhanced intensively after gadolinium administration. MRI is useful to demonstrate the lesion and to assess the damage to adjacent structures, particularly when the patient presents visual disturbances due to involvement of the cavernous sinuses.

Key words: Parasellar meningiomas. Brain tumors. Magnetic resonance imaging.
\end{abstract}

\section{INTRODUÇÃO}

Os meningiomas são os tumores cerebrais primários não-gliais mais comuns, ocorrendo numa incidência de $13 \%$ a $19 \%$ de todos os tumores opera-

* Trabalho realizado no Hospital Santa Cruz/Beneficência Portuguesa de Niterói (Scan Diagnósticos por Imagem), Niterói, RJ, Clínica X-Labs/RJ e Instituto de Pós-Graduação Médica Carlos Chagas (IPGMCC), Rio de Janeiro, RJ.

1. Professor Adjunto de Radiologia e Chefe do Serviço de Radiologia do Hospital Universitário Antônio Pedro (HUAP) da Universidade Federal Fluminense (UFF), Coordenador do Curso de Especialização em Radiologia do IPGMCC/ Beneficência Portuguesa de Niterói.

2. Professora Adjunta do Departamento de Radiologia da Universidade Federal do Rio de Janeiro (UFRJ).

3. Chefe do Serviço de Radiologia do Hospital Santa Cruz/ Beneficência Portuguesa de Niterói (Scan Diagnósticos po Imagem) e da Clínica X-Labs/RJ, Subcoordenador do Curso de Especialização em Radiologia do IPGMCC.

4. Médicos Pós-graduandos do Curso de Especialização em Radiologia do IPGMCC.

5. Professora Assistente do Departamento de Radiologia da UFF, Médica Radiologista do Hospital Santa Cruz Beneficência Portuguesa de Niterói (Scan Diagnósticos por Imagem).

Endereço para correspondência: Prof. Dr. Alair Augusto S.M.D. Santos. Avenida Geraldo de Melo Ourívio, 348, casa 31, Camboinhas. Niterói, RJ, 24346-030.

Aceito para publicação em 24/7/2000. dos. Cerca de $40 \%$ dos meningiomas ocorrem na base do crânio e $10 \%$ nas regiões parasselares, incluindo a região da asa do esfenóide, bulbo olfatório, tubérculo e diafragma selares ${ }^{(1-9)}$.

O objetivo deste trabalho é fazer uma revisão dos aspectos encontrados em exames de ressonância magnética (RM) de pacientes com meningiomas parasselares comprovados, enfocando o comprometimento das estruturas adjacentes.

\section{MATERIAL E MÉTODOS}

Foram revistos 22 casos de meningiomas parasselares avaliados com RM, em clínicas privadas nas cidades de Niterói e do Rio de Janeiro, RJ.

Os exames foram realizados utilizando-se aparelhos de 0,5 e 1,0 Tesla, através de sequiências multiplanares ponderadas em T1 (antes e após administração venosa de meio de contraste paramagnético) e em T2. Foram verificadas as queixas principais que determinaram a realização do exame de $\mathrm{RM}$, bem como os aspectos encontrados neste exame, e verificados o comprometimento do seio cavernoso, a compressão da haste hipofisária ou o componente intra-selar, a extensão supra-selar e a relação com o quiasma óptico.

\section{RESULTADOS}

Dos 22 pacientes incluídos neste trabalho, 17 eram do sexo feminino e cinco eram do sexo masculino, com a faixa etária variando dos 33 aos 71 anos (média de 52 anos), com predomínio nas sexta e sétima décadas.

As queixas principais observadas foram cefaléia e alterações visuais, tendo apenas um caso cursado com hiperprolactinemia. Nos demais casos não havia nenhuma outra alteração hormonal que evidenciasse comprometimento do eixo hipotálamo-hipofisário. 


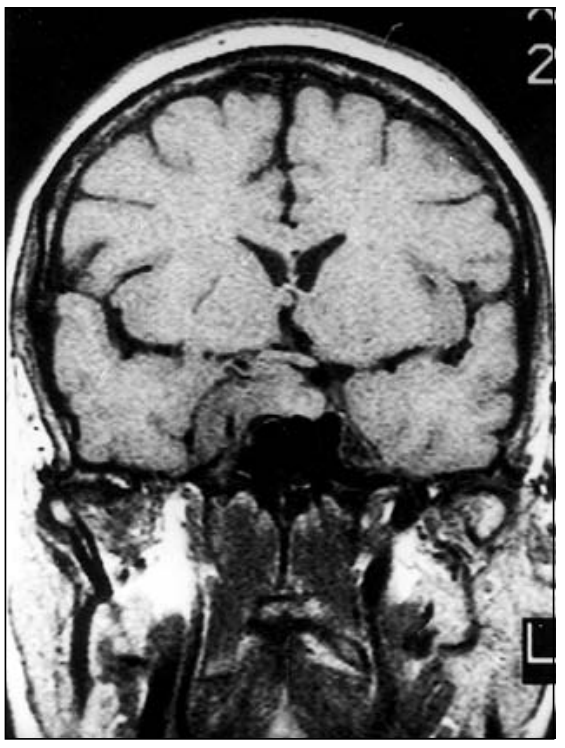

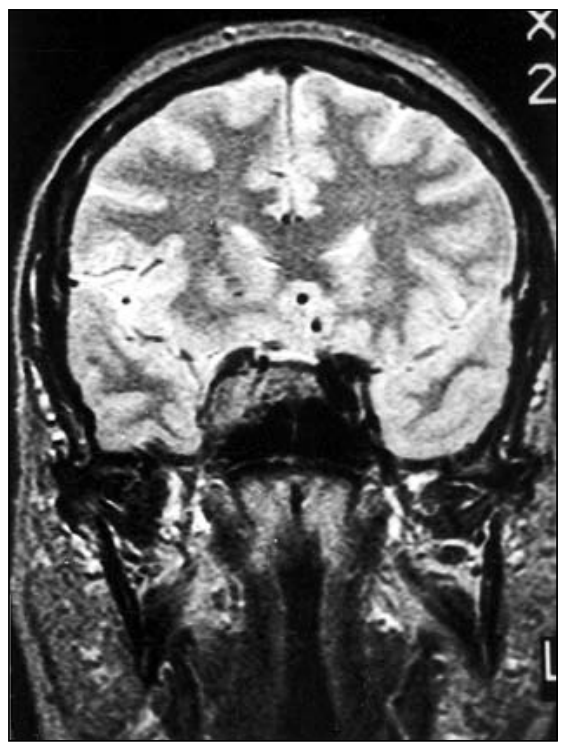

B

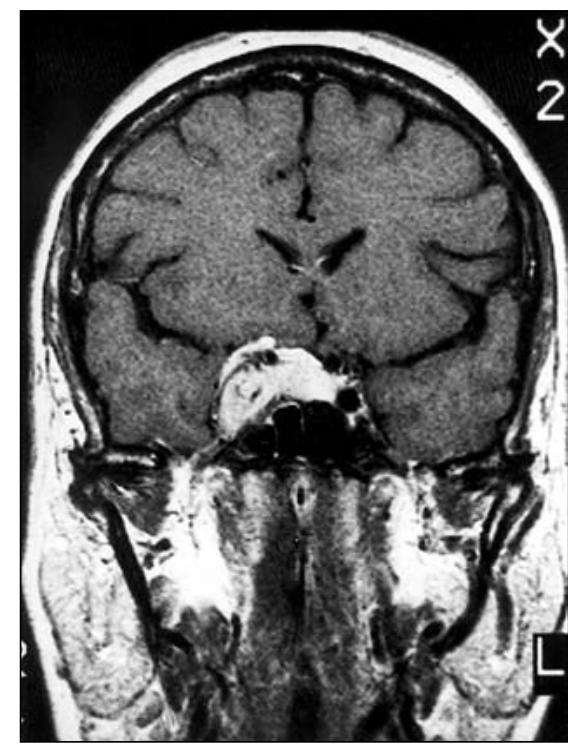

C

Figura 1. Em A, meningioma parasselar à direita, hipointenso em T1. Em B, a massa se apresenta hipointensa em T2. Em $\mathbf{C}$, após administração de gadolínio, observa-se intensa e homogênea impregnação da massa pelo meio de contraste.

$\mathrm{O}$ aspecto encontrado com mais frequiência na RM foi o de massa parasselar, hipointensa nas seqüências ponderadas em T1 e em T2, impregnando-se intensa e homogeneamente pelo meio de contraste (Figura 1).

Em 16 pacientes observou-se comprometimento do seio cavernoso, que muitas vezes encontrava-se envolvido pela massa parasselar. Em seis destes casos havia queixas de alterações visuais, que variaram da diplopia à amaurose (Figuras 2 e 3 ).

Em cinco casos havia extensão intraselar do meningioma (Figura 4).

Em relação às estruturas vizinhas, foi constatada compressão do quiasma óptico em cinco casos (Figura 5) e da haste ou da hipófise em quatro pacientes. Apenas um destes pacientes cursou com hiperprolactinemia.

\section{DISCUSSÃO}

Os meningiomas parasselares podem crescer da aracnóide na região do tubérculo selar, processo clinóide anterior, diafragma selar, plano esfenoidal, ou da dura do seio cavernoso, e podem determinar, por efeito compressivo, disfunção no eixo hipotálamo-hipofisário ${ }^{(2,6-9)}$.

São tumores comuns na idade adulta, com pico de incidência ao redor dos
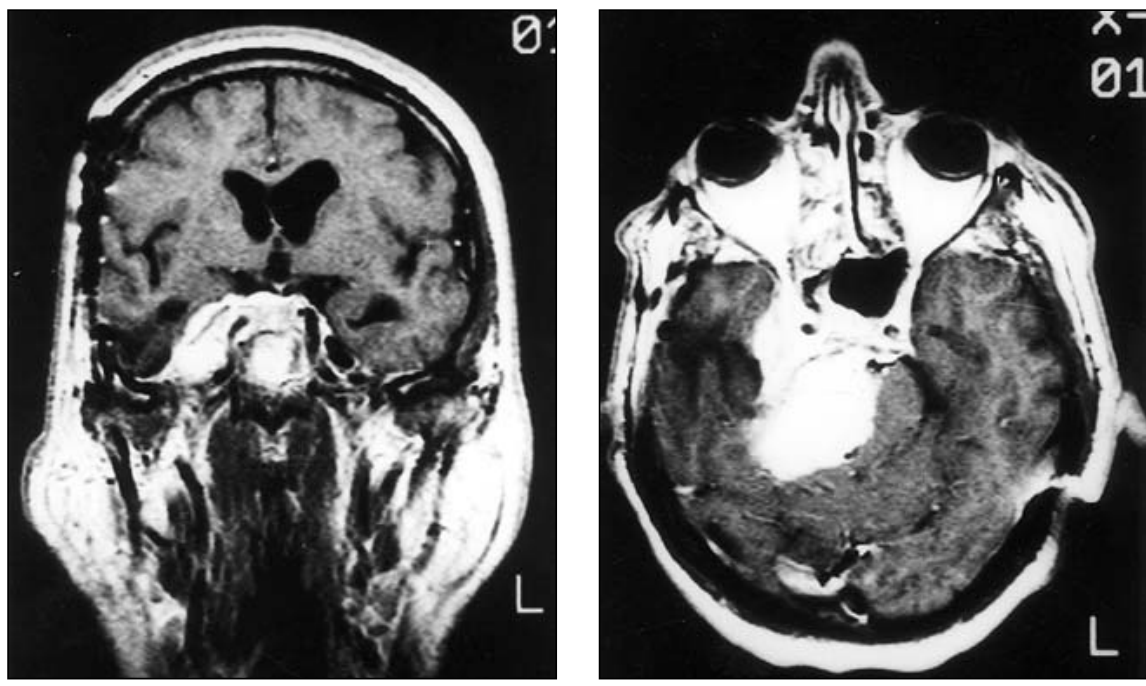

B

Figura 2. Em A, meningioma parasselar envolvendo o seio cavernoso direito. Plano coronal ponderado em T1, após gadolínio. Em B, plano axial ponderado em T1 após gadolínio.

45 anos de idade, tendo nítido predomínio no sexo feminino (cerca de $60 \%$ dos (asos) $)^{(2)}$.

Apresentam-se como massas isointensas nas seqüências ponderadas em $\mathrm{T} 1$, podendo permanecer isointensas ou hiperintensas em $\mathrm{T} 2$, e caracteristicamente impregnam-se intensamente após a administração do meio de contraste paramagnético. Podem ser evidenciados efeito de massa, espessamento da dura, fenda liquórica entre o tumor e o parênquima adjacente ${ }^{(1,10-15)}$. Nagele et al. ${ }^{(15)}$ consideram que a impregnação da dura-máter adjacente ao meningioma é sinal específico deste tipo de tumor.

O envolvimento dos vasos adjacentes pode determinar compressão da luz vascular. A angiorressonância pode ser útil no estudo da invasão de estruturas vasculares ou dos seios cavernosos pelo tumor $^{(3-5)}$.

A cefaléia, que foi um sintoma freqüente em nossos pacientes, é descrita 


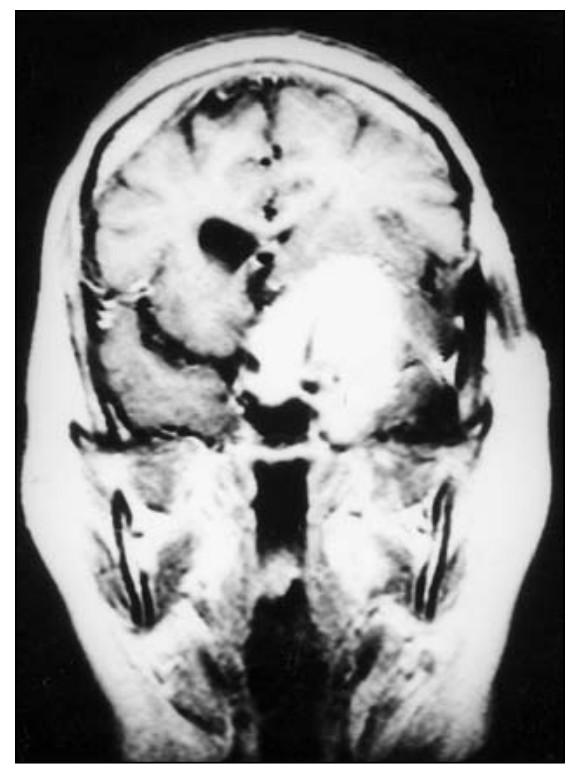

Figura 3. Meningioma parasselar esquerdo, envolvendo o seio cavernoso, englobando e desviando superiormente a artéria cerebral média homolateral.

em dois terços dos indivíduos com meningiomas suprasselares. A compressão ou mesmo a proximidade do tumor com o quiasma óptico e os nervos ópticos determinam perda progressiva da visão. $\mathrm{O}$ meningioma que invade o seio cavernoso produz paresia do nervo oculomotor ou perda sensorial da divisão oftálmica do nervo trigêmeo ${ }^{(2)}$.

No que se refere às características de sinal do meningioma na RM, quando houver sinal heterogêneo dentro do tumor pode-se sugerir a presença de calcificação, hemorragia ou degeneração cística. Nestes casos, a tomografia computadorizada (TC) é um excelente recurso para o diagnóstico diferencial, sendo fundamental para demonstrar calcificação $\mathrm{O}^{(2,5,9,12)}$.

A capacidade multiplanar da RM é superior à avaliação pela TC da localização extra-axial dos meningiomas, bem como da relação com o quiasma óptico, hipotálamo, seio cavernoso e vasos adjacentes $^{(2)}$.

Os meningiomas parasselares podem simular macroadenomas hipofisários, sendo diferenciados por apresentar multilobulações, localização excêntrica e lateral na cisterna suprasselar, extensão para o seio cavernoso e órbita, componente intra-selar pequeno e grande com-

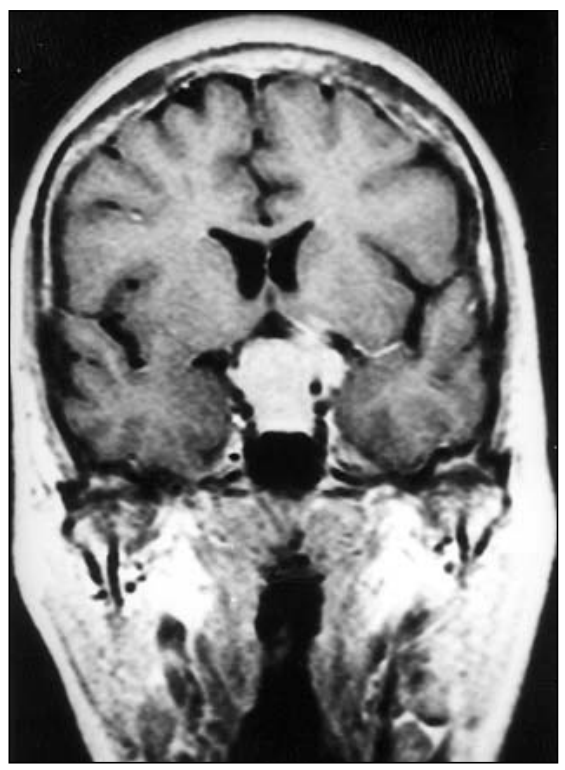

A

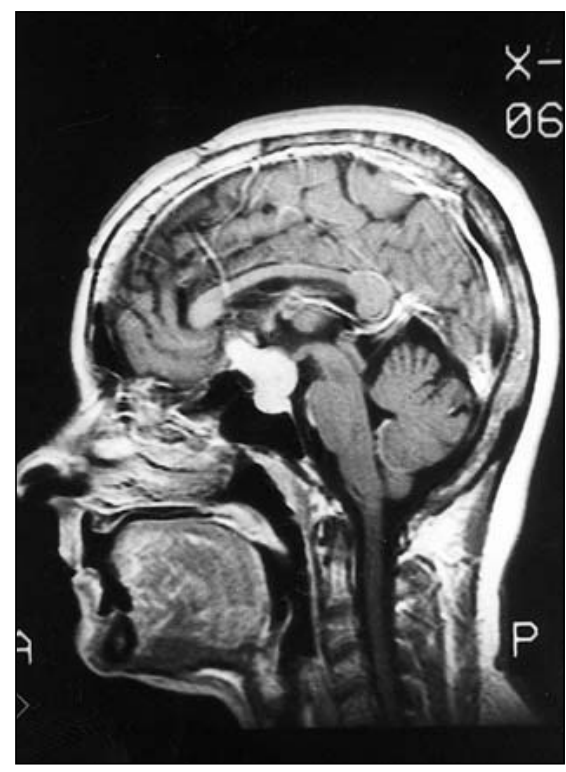

B

Figura 4. Em A, meningioma supra e intra-selar. Plano coronal ponderado em T1 após gadolínio. Em B, plano sagital demonstrando o componente intra-selar, com a hipófise preservada.
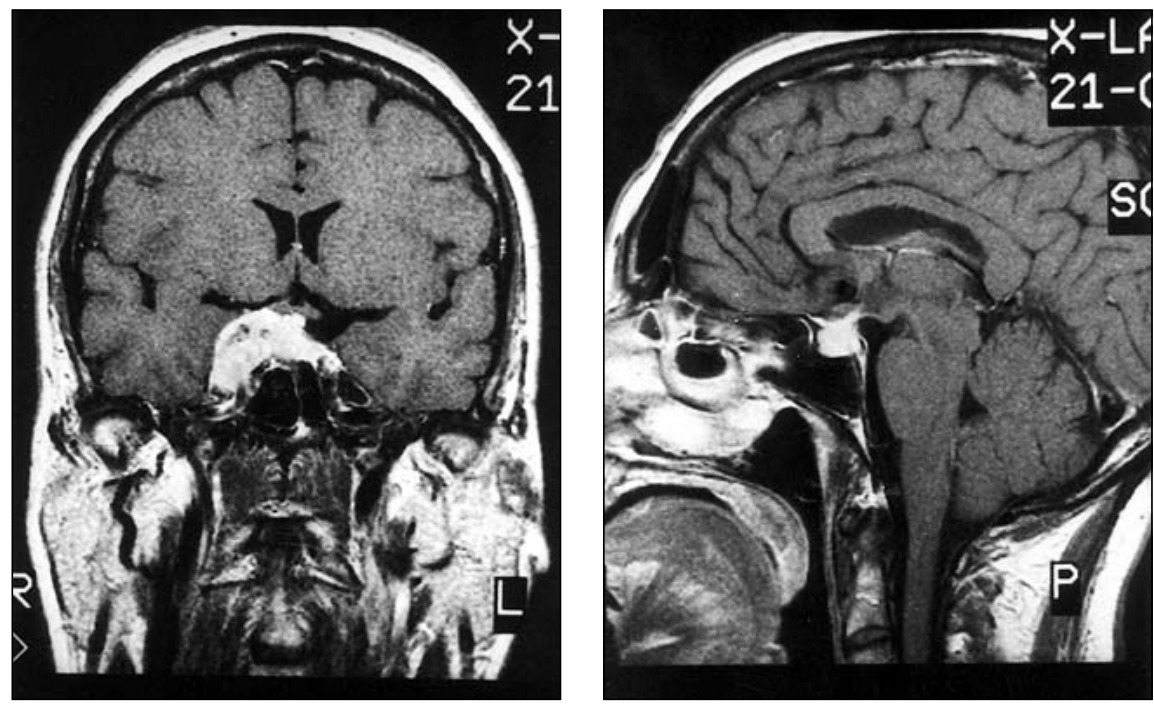

B

Figura 5. Em A, meningioma parasselar direito em contato com o quiasma óptico. Plano coronal ponderado em T1, após gadolínio. Em $\mathbf{B}$, plano sagital.

ponente extra-selar e muitas vezes sela turca de tamanho normal, podendo apresentar alterações ósseas, mais facilmente identificáveis pela $\mathrm{TC}^{(\mathbf{1 2})}$.

O diagnóstico precoce dos meningiomas parasselares é o fator mais importante no prognóstico do paciente em relação a recuperação visual, morbidade e mortalidade. A abordagem cirúrgica desses tumores é preferencialmente por via transcraniana (subfrontal), devido à possibilidade de aderências às meninges, possibilidade de fístula liquórica e outras complicações.

\section{CONCLUSÕES}

A RM permite excelente avaliação dos meningiomas parasselares, pois demonstra muito bem não apenas suas características intrínsecas, como também o envolvimento das estruturas vizinhas. 
É importante o diagnóstico o mais precoce possível, para facilitar a abordagem cirúrgica, diminuindo a morbidade e a mortalidade, buscando a recuperação visual, que é um dos sintomas mais freqüentes nesses pacientes.

\section{REFERÊNCIAS}

1. Chow SF, Andrezik JA, Hayman LA, Leeds NE. Deep tumors of the adult brain. Neuroimaging Clin N Am 1993;3:689-704.

2. Jinkins JR, Leite CC. Neurodiagnostic imaging: pattern analysis and differential diagnosis. Philadelphia: Lippincott-Raven, 1998.

3. Kucharczyk W, Montanera JW, Becker LE. The sella turcica and parasellar region. In: Atlas SW, ed. Magnetic resonance imaging of the brain and spine. 2nd ed. Philadelphia: Lippincott-Raven, 1996:871-901.

4. Chong BW, Newton TH. Hypothalamic and pituitary pathology. Radiol Clin North Am 1993;31: 1147-83.

5. Osborn AG. Diagnostic neuroradiology. St. Louis: Mosby, 1994:461-83, 649-57.

6. Elster AD. Modern imaging of the pituitary. Radiology 1993;187:1-14.

7. Felsberg GJ, Tien RD. Sellar and parasellar lesions involving the skull base. Neuroimaging Clin $\mathrm{N}$ Am 1994;4:543-60.

8. Taveras JM. Neuroradiology. 3rd ed. Baltimore: Williams \& Wilkins, 1996:627-39.

9. Breger RK, Papke RA, Pojunas KW, Haughton VM, Williams AL, Daniels DL. Benign extraaxial tumors: contrast enhancement with Gd-DTPA. Radiology 1987;163:427-9.
10. Edwards-Brown MK. Supratentorial brain tumors. Neuroimaging Clin N Am 1994;4:437-55.

11. Cecilio S, Romero PC, Dumont P, et al. Meningeoma justa-selar. Radiol Bras 1991;24:241-7.

12. Hasso AN, Bell SA, Tadmor R. Intracranial vascular tumors. Neuroimaging Clin N Am 1994;4: 849-70.

13. Kumar AJ, Lee YY, Zinreich J, Leeds NE. Imaging features of skull base tumors. Neuroimaging Clin N Am 1993;3:715-34.

14. Master L, Zimmerman RD. Imaging of supratentorial brain tumors in adults. Neuroimaging Clin N Am 1993;3:649-68.

15. Nagele T, Petersen D, Klose U, Grodd W, Opitz H, Voigt K. The "dural tail" adjacent to meningiomas studied by dynamic contrast-enhanced MRI: a comparison with histopathology. Neuroradiology 1994;36:303-7. 